\title{
Interfacial Slip Calculation of RC Beams Strengthened with FRP Plate under Symmetry Concentrated Loading
}

\author{
Jia Yang ${ }^{12, \text { a }}$ \\ ${ }^{1}$ College of Architectural and Civil Engineering, Shenyang University, Shenyang, China \\ ${ }^{2}$ The Key Laboratory of GeoenvironMental Engineering, Liaoning, China \\ ayangjia0918@163.com
}

\section{Keywords: FRP plate; Reinforced concrete; Interfacial slip}

Abstract. Debonding of fiber reinforced polymer (FRP) is a main premature failure of RC beams strengthened with FRP plate, which is mainly due to the interfacial slip between FRP and concrete beam. The interfacial slip is one of the key factors to affect the behaviors of RC beams strengthened with FRP plate. Under external loading, the relative slip occurring on the interface between FRP plate and concrete beam causes the redistribution of stress and then the bearing capacity of the member is reduced. According to elasticity theory, this study analyzes the theoretical analysis of interfacial slip of RC beams strengthened with FRP plate under symmetry concentrated loading. The analytical model is established, and the differential equation of interfacial slip of RC beams strengthened with FRP plate is established. Using the boundary conditions, the calculated formulas of interfacial slip are deduced under symmetry concentrated loading.

\section{Introduction}

In civil engineering, FRP has mainly been used to repair and to upgrade reinforced concrete structure decade ago, and is being increasingly used as an alternative to steel for strengthening RC beams. FRP plates can be bonded to the surface of RC beam through a thin adhesive layer. In this strengthening method, the performance of the FRP-concrete interface in providing an effective stress transfer is of crucial importance. At present, a large number of investigations have focused on finding possible method which used FRP sheets or plates to strengthen structures and research on its debonding failure modes and mechanical behavior ${ }^{[1-6]}$. But the research about the interfacial slip of the FRP-concrete interface is relatively less. Under external loading, the relative slip occurred on the interface between FRP plate and concrete causes the redistribution of stress and then influences mechanical behavior of component. Therefore, this paper studies on the slip of FRP-concrete interface. Based on the analytical model, the differential equation of interfacial slip is established. And the calculated formulas of interfacial slip are deduced under symmetry concentrated loading.

\section{Analytical Model}

\section{Governing Differential Equation}

A differential section $(d x)$ is cut out from the RC beams strengthened with FRP plate. The composite beam is made of three materials: RC concrete, adhesive layer and FRP plate. In the present analysis linear elastic behavior is assumed for all the materials. The adhesive is considered only in transferring the stresses from the concrete to the FRP reinforcement and the stresses in its layer do not change through the direction of the thickness. 
In this study, the following assumptions are made: 1. The beam, adhesive, and FRP plate are having elastically and linearly. 2. Stresses in the adhesive layer do not change with the thickness. 3. Deformations of FRP plate and concrete beam are due to bending moments, axial and shear forces. 4. The curvatures of FRP plate and concrete beam are assumed to be the same. 5. The axial force, bending moment and shear force of adhesive layer are ignored. So the adhesive layer is exposed only to the interfacial shear and normal stresses. Adhesive layer is mainly transmitted shear force and shear deformation.

The interfacial shear stress is denoted $\tau$. The constitutive equations for the interface can be expressed as follow.

$$
\tau=f(\delta)
$$

In Eq. 1, the interfacial slip $\delta$ is defined as the relative displacement between the FRP plate and concrete beam, and then $\delta$ can be written as below.

$$
\delta=u_{f}(x)-u_{c}(x)
$$

Where $u_{c}(x)$ and $u_{f}(x)$ are the longitudinal displacements at the base of concrete beam and the top of FRP plate respectively.

After differentiating, the Eq. 2 can be expressed in terms of mechanical strain of concrete beam and FRP plate as follow.

$$
\frac{d \delta}{d x}=\varepsilon_{f}(x)-\varepsilon_{c}(x)
$$

Where $\varepsilon_{c}(x), \varepsilon_{f}(x)$ are the strain at the bottom of concrete beam and the top of FRP plate respectively.

The strain formulas of concrete beam and FRP plate are given as follow.

$$
\begin{aligned}
& \varepsilon_{c}(x)=\frac{M_{c}(x) y_{1}}{E_{c} I_{c}}-\frac{N_{c}(x)}{E_{c} A_{c}} \\
& \varepsilon_{f}(x)=-\frac{M_{f}(x) y_{2}}{E_{f} I_{f}}+\frac{N_{f}(x)}{E_{f} A_{f}}
\end{aligned}
$$

Where $E_{c}, E_{f}, I_{c}, I_{f}$ are elastic modulus, inertia moments of RC beam and FRP plate respectively; $A_{c}, A_{f}$ are cross-sectional areas of RC beam and FRP plate respectively; $M_{c}(x), M_{f}(x), N_{c}(x), N_{f}(x), V_{c}(x), V_{f}(x)$ are the bending moment, axial and shear forces in each adherend while $y_{1}$ and $y_{2}$ are the distances from the bottom of RC beam and the top of FRP plate to their respective centroid.

The following equilibrium equations are established as below.

$$
\begin{aligned}
& \frac{d M_{f}(x)}{d x}=V_{f}-\tau b_{f} y_{2} \\
& \frac{d M_{c}(x)}{d x}=V_{c}-\tau b_{f} y_{1} \\
& \frac{d N_{c}(x)}{d x}=\frac{d N_{f}(x)}{d x}=\tau b_{f}
\end{aligned}
$$



Eq.9.

Substituting Eqs. 4-8 into Eq.3, and get derivative, then the differential equation refers with

$$
\begin{aligned}
& \frac{d^{2} \delta}{d x^{2}}-\alpha^{2} f(\delta)+\beta V(x)=0 \\
& \text { Where } \alpha^{2}=b_{f}\left[\frac{1}{E_{f} A_{f}}+\frac{1}{E_{c} A_{c}}+\frac{\left(y_{1}+y_{2}\right)^{2}}{E I}\right], \beta=\frac{y_{1}+y_{2}}{E I}, E I=E_{f} I_{f}+E_{c} I_{c} \text { and } V(x)=V_{f}+V_{c} .
\end{aligned}
$$

\section{Interfacial bond-slip model}

Various bond-slip models have been considered in previous work. The model of stress-slip relationship is considered to be possible in representing the nonlinear interfacial behavior.

The mathematical expression is as follow.

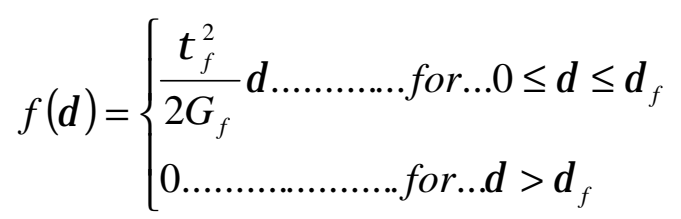

Where, $\tau_{f}$ is the local bond strength, and $\delta_{f}$ is the maximum slip, and $G_{f}$ is the interfacial fracture energy. Substituting Eq.10 into Eq.9, when $0 \leq \delta \leq \delta_{f}$, the differential equation of interfacial slip refers with Eq.11.

$$
\begin{aligned}
& \frac{d^{2} \delta}{d x^{2}}-\omega^{2} f(\delta)+\beta V(x)=0 \\
& \text { Where } \omega^{2}=\frac{b_{f} \tau_{f}^{2}}{2 G_{f}}\left[\frac{1}{E_{f} A_{f}}+\frac{1}{E_{c} A_{c}}+\frac{\left(y_{1}+y_{2}\right)^{2}}{E I}\right] .
\end{aligned}
$$

\section{Calculation formulas}

The differential equation of interfacial slip has different solutions under different loads. Under the effect of symmetry concentrated loading, the calculation formulas of interfacial slip are established.

Under symmetry concentrated loading $\mathrm{P}$ (beam length is $L$, length of FRP plate is $l_{f}$ ), the beam can be divided into bending-shear part and pure bending part, and the general solutions of differential equation can be established respectively. The solutions to differential equation of interfacial slip refer with Eq.12-13.

On bending-shear part, the general solution of differential equation refers with Eq.12.

$$
\delta_{1}=A_{1} e^{\omega x}+A_{2} e^{-\omega x}-\frac{\beta P}{\omega^{2}}
$$

On pure bending part, the general solution of differential equation refers with Eq.13.

$$
\delta_{2}=A_{3} e^{\omega x}+A_{4} e^{-\omega x}
$$

Where $A_{1}$, and $A_{2}$, and $A_{3}$, and $A_{4}$ are constant.

The solutions to differential equations of interfacial slip can be obtained by using the following boundary conditions. The boundary conditions are respectively: 
1. when $x=0$, there is $\delta_{2}=0 ; 2$.when $x=0.5 l_{0}$, there are $\delta_{1}=\delta_{2}$ and $\frac{d \delta_{1}}{d x}=\frac{d \delta_{2}}{d x} ; 3$. when $x=0.5 l_{f}$, there is $\frac{d \delta_{1}}{d x}=-\frac{P\left(L-l_{f}\right)\left(y_{1}+y_{2}\right)}{2 E I}=-\delta_{a}$.

The formulas of the constants respectively refer with Eq.14, Eq.15 and Eq.16 and Eq.17.

$$
\begin{aligned}
& A_{1}=\frac{\beta P\left(e^{-\frac{\omega l_{0}}{2}}+e^{\frac{\omega l_{0}}{2}}\right)-2 \omega \delta_{a} e^{\frac{\omega}{2} l_{f}}}{2 \omega^{2}\left(1+e^{\omega l_{f}}\right)} \\
& A_{2}=\frac{\beta P\left(e^{-\frac{\omega l_{0}}{2}}+e^{\frac{\omega l_{0}}{2}}\right) e^{\omega l_{f}}+2 \omega \delta_{a} e^{\frac{\omega}{2} l_{f}}}{2 \omega^{2}\left(1+e^{\omega l_{f}}\right)} \\
& A_{3}=\frac{\beta P\left(e^{\frac{\omega l_{0}}{2}}-e^{\omega l_{f}-\frac{\omega l_{0}}{2}}\right)-2 \omega \delta_{a} e^{\frac{\omega}{2} l_{f}}}{2 \omega^{2}\left(1+e^{\omega l_{f}}\right)} \\
& \left.A_{4}=-\frac{\beta P\left(e^{\frac{\omega l_{0}}{2}}-e^{\omega l_{f}-\frac{\omega l_{0}}{2}}\right)-2 \omega \delta_{a} e^{\frac{\omega}{2} l_{f}}}{2 \omega^{2}\left(1+e^{\omega l_{f}}\right.}\right)
\end{aligned}
$$

\section{Conclusions}

Based on the analytical model, the differential equation of interfacial slip of RC beams strengthened with FRP plate is established. And the calculated formulas of interfacial slip are deduced under symmetry concentrated loading. The calculation formulas of interfacial slip are rational in theory, but the applicability of the calculation formulas needs more experiments to verify.

\section{References}

[1] Lianguang Wang. Prestressed Composite Structures of Steel and Concrete. Beijing Science Press (2009) in press. (In Chinese)

[2] Teng J. G, Yuan H. FRP-to concrete interface between two adjacent cracks: Theoretical model for debonding failure. International Journal of Solids and Structures, Vol. 43(2006), p. 5750.

[3] Tounsi Abdelouahed, Improved theoretical solution for interfacial stresses in concrete beams strengthened with FRP plate. International Journal of Solids and Structures 43 (2006), 4154-4174

[4] Zhishen Wu, Hong Yuan, and Hedong Niu. Stress Transfer and Fracture Propagation in Different Kinds of Adhesive Joints. JOURNAL OF ENGINEERING MECHANICS, MAY 2002.128:562-573.

[5] Fangliang Chen, Pizhong Qiao. Debonding analysis of FRP-concrete interface between two balanced adjacent flexural cracks in plated beams. International Journal of Solids and Structures, Vol. 46 (2009), 2618-2628.

[6] Lin Xinyan, Cao Shuangyin, Huang Fengxia. Analysis on bond shear stress of concrete ties strengthened with FRP. Journal of Building Structures(Supplementary Issue), p.779-782. (In Chinese) 